\title{
Anticipatory vibrotactile cueing facilitates grip force adjustment
}

\author{
Shogo Okamoto \\ Department of Mechanical Sci. and Eng. \\ Nagoya University, Nagoya, Japan
}

\author{
Michael Wiertlewski \\ Department of Mechanical Eng. \\ Northwestern University, Evanston, USA
}

\author{
Vincent Hayward \\ UPMC Univ Paris 06, Institut des \\ Systèmes Intelligents et de Robotique,
}

\begin{abstract}
Human grip forces are automatically adjusted upon occurrence of an external disturbance experienced by an object that is held by a thumb and index finger. We investigated some of the cues that may be used by the brain to perform rapid grip restabilization. To this end we ask subjects to grip and hold an instrumented and actuated parallelepiped-shaped handle between the index finger and the thumb. Under computer control, the handle could be jerked from the still grip and could independently provided vibration of 250 or $100 \mathrm{~Hz}$ to the gripping fingers. We found that the latency of the motor corrective action was $139 \mathrm{~ms}$ on average, but when a vibrotactile stimulation was applied $50 \mathrm{~ms}$ before the application of the pulling force, the latency was reduced on average to $117 \mathrm{~ms}$. The average latency of the conscious response to the vibrotactile stimuli was $230 \mathrm{~ms}$, suggesting that vibrotactile stimulation was capable of influencing the reflex action.
\end{abstract}

\section{INTRODUCTION}

Grip force adjustment is automatically performed during manipulation. This adjustment is largely unconscious and is particular useful in the case of rapid, unexpected variations in the loading and unloading of the grip [7]. The result is an automatic stabilization of the grip preventing slip, yet avoiding overly large grip forces to be employed. To achieve this function, it is likely that several populations of mechanoreceptors provide information to multiple spinal and supraspinal sensorimotor loops operating at different levels of the nervous system. Among these populations, mechanoreceptors associated with cutaneous inputs play an important role since digital anesthesia results in substantial attenuation of the gripping sensorimotor corrective behavior $[5,2,1]$, where an increase of the grip force could be interpreted as a strategic response of the nervous system to weakened inputs $[3,14]$. Also, vibrotactile stimulation to the finger pads affect the grip force when the gripped object experiences perturbation [12]. It can be concluded from these studies that cutaneous inputs are able to provide early-warning signals, in advance of the catastrophic failure of a grip. Grip force adjustment is an intriguing form of behavior that one would desire to replicate for robotic [16, 20, 10, 17], and rehabilitation purposes [8,9].

The shortest latency between the onset of a load and grip force adjustment was found to be approximately $70 \mathrm{~ms}[2,7]$ but according to the loading conditions, this value can vary up to $170 \mathrm{~ms}$ [6]. We focus here on grip force adjustment from the aspect of interface design for a more stable human grasp. Prior studies have looked at the effect of added stimulation during the change of grip of load $[12,15]$. From these studies it can be concluded that additional stimulation can modify the subsequent gripping behavior.

Here, our hypothesis is that stimulation of the fingertips can shorten the latency of the onset of grip force adjustment. To test this hypothesis we organized experimental conditions where the fingertips could be cued ahead of the slip-inducing, sudden load increase. In an attempt to segregate the mechanical cues that could be de-
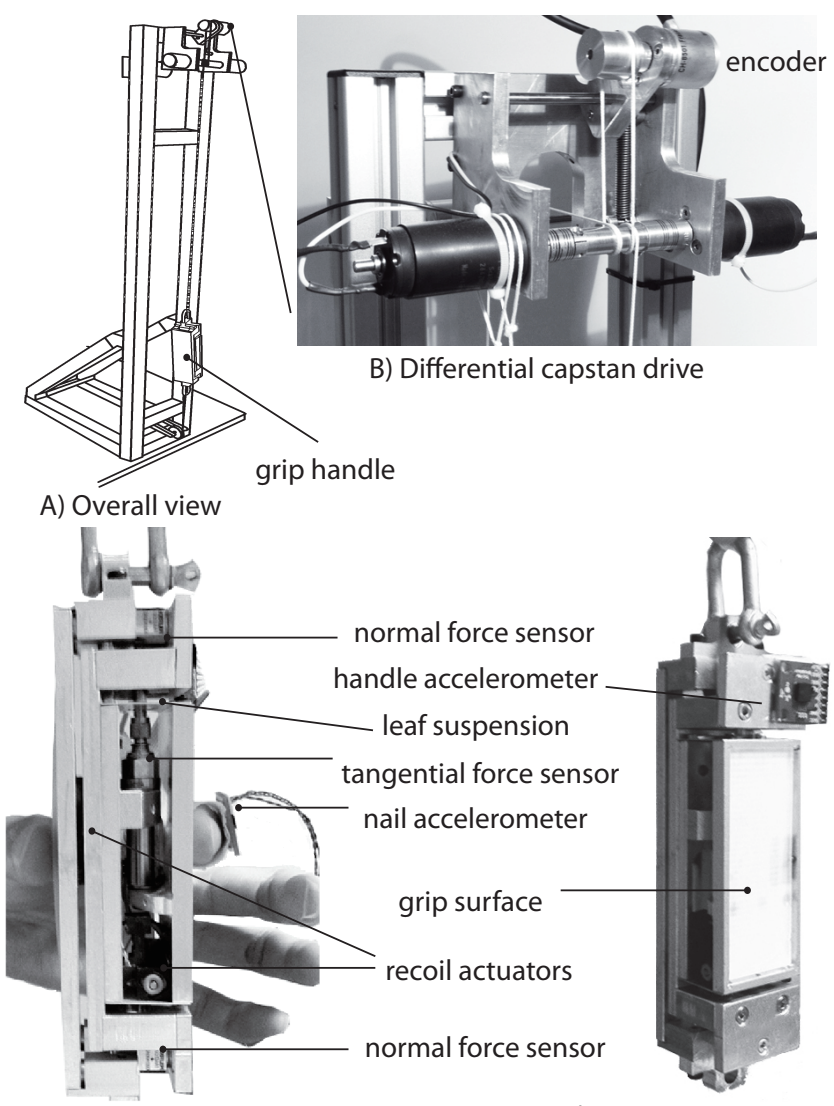

C) Side view of grip handle

D) Perspective view

Figure 1: Apparatus including an instrumented grip handle and a pulling mechanism. A: Overall view. B: Differential capstan drive with two motors. C: Side view of the grip handle. D: Perspective view of the grip handle.

terminant in provoking a grip adjustment, we also measured the fingertip bulk deformation during behavior.

Finally, this is one of the paired articles on the study of vibrotactile stimuli and their effects on grip force adjustment. Another article mainly discusses vibrotactile cues induced by slippage [19].

\section{Material and Methods}

\subsection{Apparatus}

Figure 1 shows the experimental setup. The main component was a grip handle to be held between the thumb and the index fingers and driven by two DC motors (2642W012CR, Faulhaber) in tandem using a differential capstan mechanism. Such mechanisms can achieve arbitrarily large bi-directional traction for an arbitrarily low pre-tension in the cable, thus insuring smooth operation and minimal friction losses [4]. The present system provides $20 \mathrm{~N}$ of continuous pulling force for less than $0.1 \mathrm{~N}$ of friction. Position was 
measured by a potentiometer (Baumer, BTIV 24S 16.24K, resolution: $0.0146 \mathrm{~mm}$ ). The motors were commanded and the data was collected through a multifunction DAQ card at a sampling rate of $10 \mathrm{kHz}$ (PCI-6221, National Instruments).

The grip handle had three uni-axial force sensors organized as follows. The normal grip force component was measured by two force sensors mounted in the load paths between the two plates at each end of the handle (X-axis: 9313AA1, Kistler). The plate interacting with the index included a leaf suspension mechanisms that separated out the tangential component of the interaction force which was measured by a high resolution, high bandwidth force sensor (Z-axis: 9217A, Kistler). The fingers were in contact with smooth, sanded finish plastic surfaces.

Two accelerometers were used to measure the deformations of finger pad during loading. The acceleration of the grip handle was measured by a miniature solid state accelerometer (MMA7260QT, Freescale Semiconductor, nominal bandwidth: $350 \mathrm{~Hz}$ ) and the acceleration of the nail of the index finger measured by a second miniature accelerometer (ADXL300, Analog Devices, nominal bandwidth: $1600 \mathrm{~Hz}$ ). The differential acceleration gave a measure of the deformation of the finger, bulk strain rate by a first step of integration and bulk deformation by a second step of integration. Because of the short time window and in the absence of slip, a precise estimate of the finger can be obtained this way. Moreover, full slip can be detected when the rate and amplitude of the deformation exceed thresholds.

Two recoil-type actuators (Haptuator Mark 2, Berrezag Amir) could vibrate the grip handle. They were mounted orthogonally to loads paths of the force sensors to minimize interference with the force measurements.

\subsection{Participants, Task, and Stimuli}

Five volunteer participants, which excluded the authors, from the research staff of the institution joined the experiments. During the experiments, they wore headphones playing pink noise to mask out the faint sounds emitted by the vibration actuators and by the pulling motors.

The participants sat on a chair and held the grip handle between their thumb and index fingers. They were instructed to hold the grip handle in a relaxed manner with a natural grip sufficient to maintain it under gravity loading. They were asked to "hold the grip handle such that you do not lose it, and respond to the vibrations as quickly as possible."

In some test conditions, after a randomized a time period (2-5s), an impulsive command could be sent to the motors and the grip handle was rapidly accelerated upward. The result of this acceleration was a sudden increase of the load applied to the fingers, see Fig. 2 for a typical example. The interaction force peaked on average at $5 \mathrm{~N}, 130 \mathrm{~ms}$ after the onset of the command sent to the motors and the force rate reached $38.5 \mathrm{~N} / \mathrm{s}$ on average, reflecting the dynamics of the handle-grip mechanics. In certain test conditions, vibrotactile stimulation was applied to the participants' thumb and index fingers. The magnitude of vibration was approximately $\pm 10 \mathrm{~m} / \mathrm{s}^{2}$.

\subsection{Testing Conditions}

We prepared for four conditions that differed according to the loading and vibrotactile stimuli. The participants experienced these four conditions in practice phases that lasted a few minutes. During the main task, each condition was presented ten times. In total, for individual participant, forty trials were performed in a randomized order. After each trial, the participants released the grip handle and relaxed. Upon visual cueing, the participant gripped the grip handle and conducted the next trial.

Loading only (Condition 1). The load was suddenly increased as described previously.
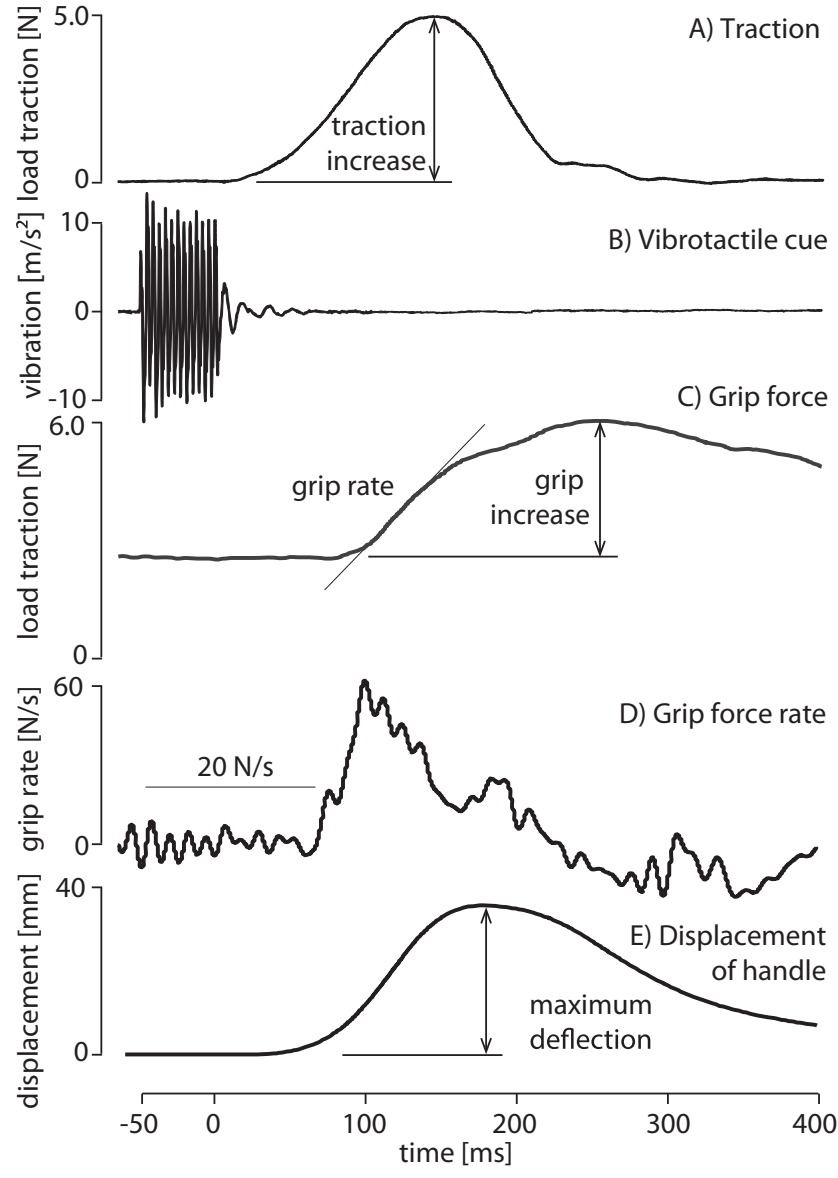

Figure 2: Time course of the loading stimulus, vibrotactile stimulus, motor response and net grip handle displacement. A: Traction loading force component reaching a maximum, typically at $130 \mathrm{~ms}$ after the torque command to the motors. B: Vibrotactile stimulus. C and D: grip force response and rate. E: Net displacement of the grip handle, reaching a maximum at a different time from the load due to complex biomechanical dynamics coupled to the inertial dynamics of the motor/handle subsystem.

Vibrotactile stimulation only at $250 \mathrm{~Hz}$ (Condition 2). Vibrotactile stimulation at $250 \mathrm{~Hz}$ was produced as described previously, but not followed by sudden loading. The aim of this condition was to obtain and estimate the latencies of conscious response to vibrotactile stimulation.

Load and vibrotactile stimuli at $250 \mathrm{~Hz}$. (Condition 3). Vibrotactile stimulation at $250 \mathrm{~Hz}$ was presented $50 \mathrm{~ms}$ before the onset of loading to the participants. The aim of this condition was to observe the effects of vibrotactile stimuli on grip force adjustment.

Load and vibrotactile stimuli at $100 \mathrm{~Hz}$. (Condition 4). This condition was the same as Condition 3 with the difference that the vibration frequency was $100 \mathrm{~Hz}$. The aim was to investigate whether there was some frequency specificity visible in the motor response given the likely contribution of the Pacinian receptor population.

Follow-up trials (Condition 5). After forty trials in Conditions 1-4, we repeated tests in Condition 2 for ten trials in order to evaluate the conscious responses to vibrotactile stimuli. In these followup trials, the participants were informed that only vibrotactile cues would be presented to them, and had to respond to them as quickly as possible. 
Table 1: Averages and standard deviations of three parameters among all participants. 'Id' and 'vib' indicate load and vibrotactile cue, respectively.

\begin{tabular}{c|l|c|c|c}
\hline \multicolumn{2}{l|}{} & $\begin{array}{c}\text { Latency } \\
{[\mathrm{ms}]}\end{array}$ & $\begin{array}{c}\text { Grip inc- } \\
\text { rease [N] }\end{array}$ & $\begin{array}{c}\text { Disp. grip } \\
\text { handle [mm] }\end{array}$ \\
\hline \multirow{2}{*}{} & 1 (ld only) & $139 \pm 30$ & $8.76 \pm 4.21$ & $32.4 \pm 17.0$ \\
2 (vib only) & $229 \pm 97$ & - & - \\
\multirow{0}{*}{} & 3 (ld + vib) & $116 \pm 23$ & $9.91 \pm 4.55$ & $32.0 \pm 15.0$ \\
& 4 (ld + vib) & $117 \pm 24$ & $10.5 \pm 4.88$ & $30.5 \pm 13.8$ \\
\cline { 2 - 5 } & 5 (vib only) & $172 \pm 55$ & - & - \\
\hline
\end{tabular}

\subsection{Data Analysis}

Several data can be extracted from these experiments. Figure 2 shows typical records of the time course of events, including traction loading, grip force response and the net displacement of the grip handle. If the command is applied at time, $t=0$, approximately $100 \mathrm{~ms}$ later, the onset of grip force adjustment was typically observed. Using these data, three types of parameters were evaluated for each trial. The largest and smallest values of these parameters among those of each condition of individual participants were automatically pruned, and then the rest were used for statistics.

The latency between traction force and the grip force adjustment was defined at the time difference of their respective onsets in Conditions 1, 3, and 4. For conditions 2 and 5, this value was defined as time difference between the end of the vibrotactile cue and the onset of grip force adjustment. In Conditions 3 and 4, the end of the vibrotactile cue and the beginning of the rise in traction force coincided, as a result of the very high stiffness of the mechanical transmission between the motors and the handle. We are then allowed to compare the responses among conditions using the same temporal baseline. The derivative of grip force signals was computed by finite difference after FFT filtering with a cut-off frequency of $100 \mathrm{~Hz}$. An example can be seen in Fig. 2. The threshold as $20 \mathrm{~N} / \mathrm{s}$ was used to mark the beginning of grip force change. This threshold is approximately two to three times the noise levels. For some trials, the grip force change rate did not reach this threshold level. These trials were excluded from the reports.

The increase in grip force was the difference in grip force between its maximum and the average of the last $100 \mathrm{~ms}$ before the onset of the traction force, or the end of the vibrotactile stimulation. The grip force values were filtered in the way same as just described. This value is known to depend on conditions of perturbative loads, and has been used as an important parameter to characterize grip force adjustment. Also, the magnitude of grip force is directly related to stable grasping.

The maximum deflection was defined as the difference in the position of the grip handle between the highest value after the force stimulus and the average of $100 \mathrm{~ms}$ before the onset of traction. Unfiltered position signals from the encoder were used to calculate this value. It is also an important parameter to evaluate the success of grip force adjustment. Smaller values indicate a more successful recovery from disturbance.

\section{Results}

\subsection{Latency of grip force adjustment}

As a whole, the latencies between the pulling force and grip force adjustment, vibration stimulation only (Condition 2) clearly recorded the largest values among four conditions. Condition 2 was, then, followed by the loading only condition (Condition 1) that was potentially significantly larger than the condition with combined loading and vibration at $250 \mathrm{~Hz}$ and at $100 \mathrm{~Hz}$ (Conditions 3 and 4). The latencies in Conditions 3 and 4 were nearly indistinguishable.

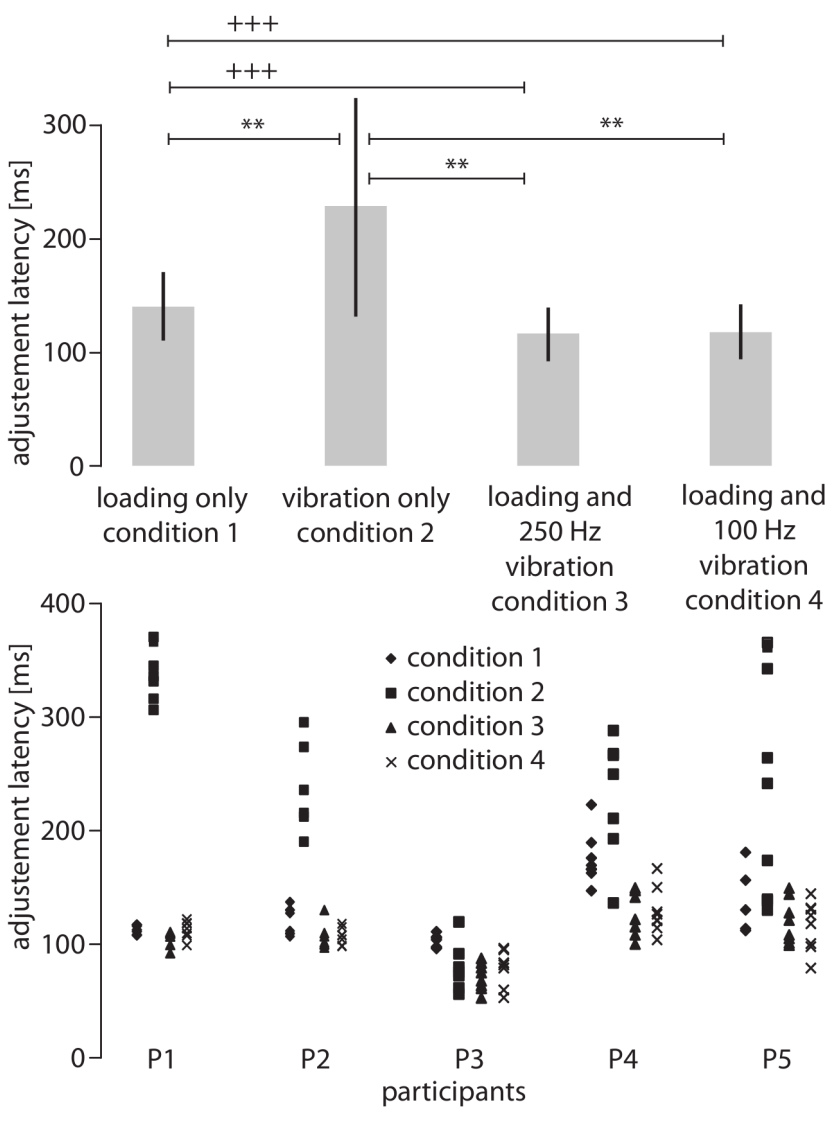

Figure 3: Latency between pulling force and grip force adjustment. Averages and standard diviations among trials. Top) Entire trials. Bottom) By participants P1-P5. Dots correspond to conditions 14 from left to right for individuals. ${ }^{* *}: p<0.01$ by Tukey test. ${ }^{+++ \text {: }}$ $p<0.001$ by $t$-test.

Figure 3 (top) and Table 1 show the average and standard deviation values among all trials by condition. Figure 3 (bottom) shows the latencies for each trial by participants. The latencies of the vibration only condition (Condition 2) were clearly larger than those of the other conditions with an average value of $229 \mathrm{~ms}$ hence of $279 \mathrm{~ms}$ from the onset of the cue. The latencies of load only condition 1 (Condition 1) recorded the second highest values. The other conditions followed.

The Tukey test showed significant differences between Condition 2 and the others (conditions 1 and 2: $q(3,145)=7.22$, $p<0.01$, conditions 2 and 3: $q(3,145)=9.34, p<0.01$, conditions 2 and 4: $q(3,145)=9.23, p<0.01)$. We also applied pairwised $t$-tests to the pairs of conditions, which additionally indicated significant differences between conditions 1 and $3(t(73)=3.89$, $p<0.001$, two-tailed) and conditions 1 and $4(t(73)=3.59, p<$ 0.001, two-tailed). As shown in Fig. 3 (bottom), these trends were observed for all individuals. For P3, the latencies of condition 2 seem smaller than those of condition 1 . However, note these are conservative estimates and that we should add $50 \mathrm{~ms}$ to the values of condition 2 when comparing the latencies of conditions 1 and 2 .

\subsection{Increase in grip force}

In term of the increases in grip forces, no statistically significant differences were found among conditions. However, as a weak trend, the presence of vibration seemed to provoke a larger increase of grip force of about 1 to $2 \mathrm{~N}$ (Condition 3 and 4). Fig. 4 (top) and Table 1 show the average and standard deviation values of all trials 


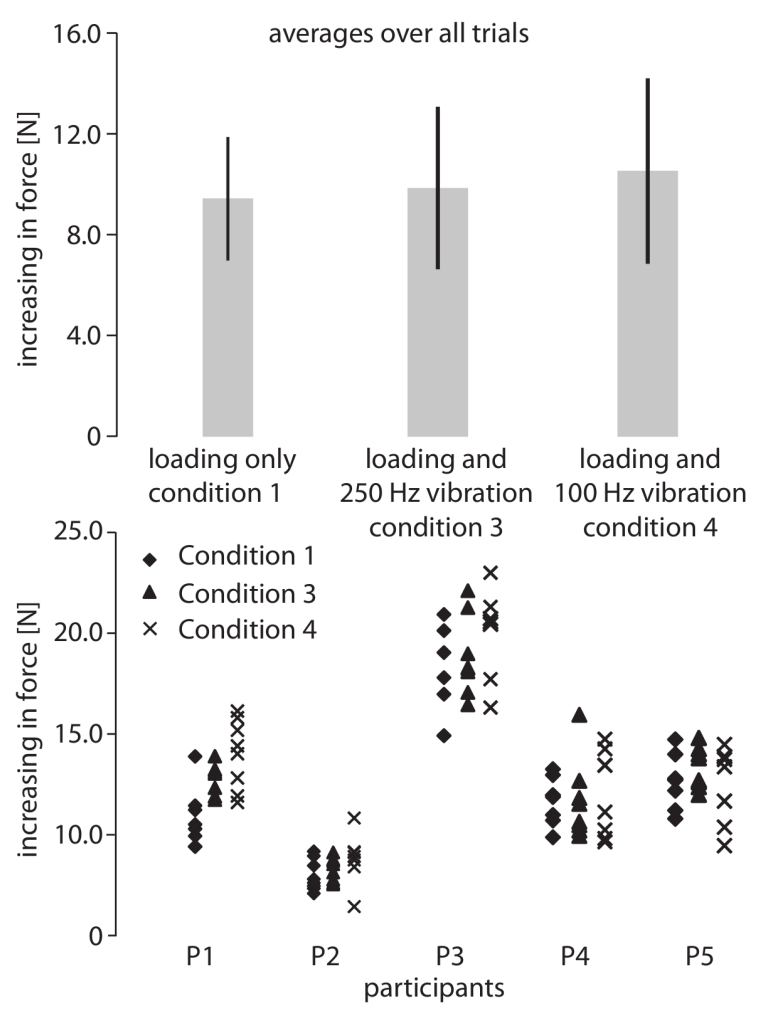

Figure 4: Increase in grip force. Averages and standard diviations among trials. No statistically significant differences were exhibited among conditions.

by conditions. Fig. 4 (bottom) shows the results by individual participants. The vibration only condition (Condition 2) was excluded. On average, the increases in grip forces were the smallest in the load only condition (Condition 1), followed by the load combined with vibrations conditions (Conditions 3 and 4).

As shown in Fig. 4 (bottom), for three participants among five (P1-P3), these trends held. For P5, the increases in grip force were highest under condition 3 , and those of conditions 1 and 4 were approximately equal. For $\mathrm{P} 4$, condition 4 exhibited the highest values, and conditions 1 and 3 recorded similar values on average.

\subsection{Handle Displacement}

Table 1 shows the averages and standard deviations of the displacements of grip handle. These values did not significantly vary among the conditions.

\subsection{Detailed analysis of the results for one participant}

Fig. 5 shows the average time course of the grip force, grip force rate, and the displacement of the grip handle for participant P5. These profiles are the averages of ten trials for each condition. Data from each trial referenced to zero time and displacement. As shown in the top panel figure, for this participant, the increase in grip force was the largest in the condition where the loading and a vibration at $250 \mathrm{~Hz}$ were both present (Condition 3). This increase value was followed by the values obtained in the condition of loading only (Condition 1) and loading and vibrations at $100 \mathrm{~Hz}$ (Condition 4). The latencies (top and middle panels), obtained with loading plus vibration, were the shortest but comparable (conditions 3 and 4), followed by the loading only condition and lastly with vibrations only (Condition 2). As shown in the bottom of Fig. 5, while there was no unified tendency among participants, for this participant the

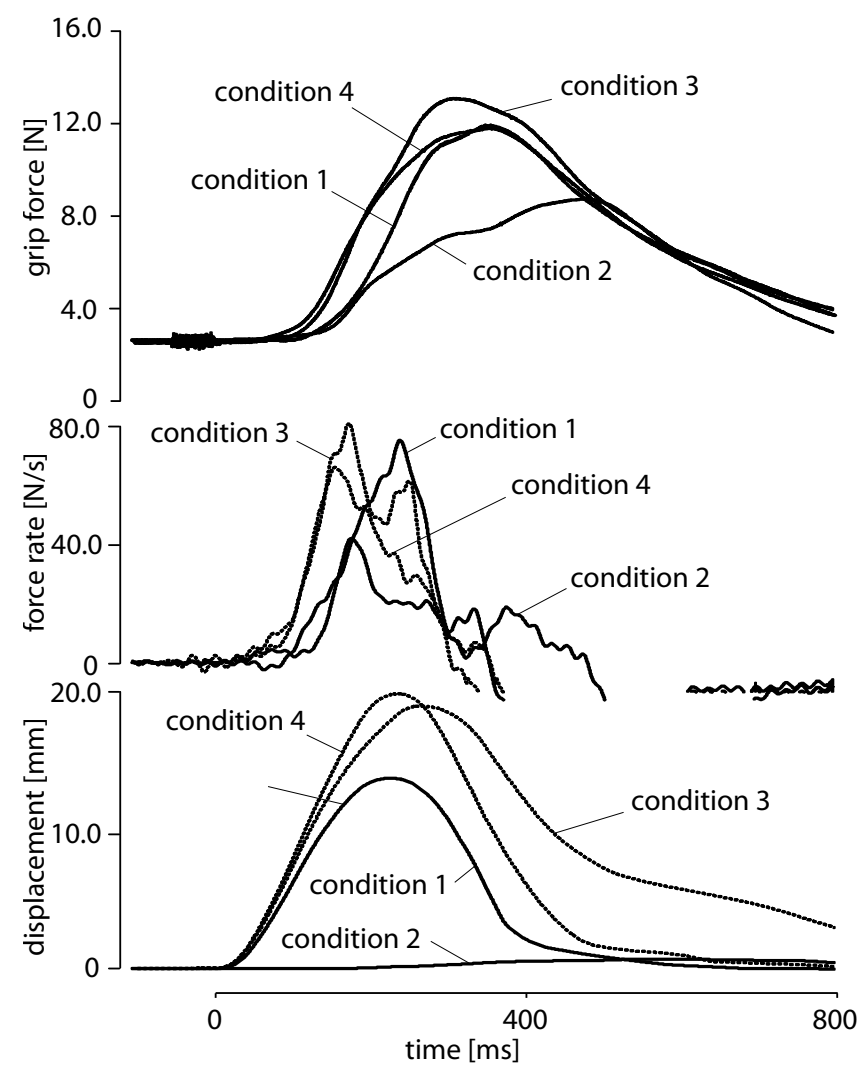

Figure 5: Average results of one participant. Top) Grip force. Middle) Derivative of grip force. Bottom) Position of the grip handle. Condition 1 is load stimulus only. Condition 2 is vibrotactile stimulus only. Condition 3 is load + vibrotactile stimuli $(250 \mathrm{~Hz})$. Condition 4 is load + vibrotactile stimuli $(100 \mathrm{~Hz})$.

displacements under Condition 4 were the largest. As to the displacement of grip handle, while there was no unified tendencies observed between the participants, for this participant, the presence of vibrations made a noticeable difference.

\subsection{Finger Pad Deformation}

Some interesting data was obtained from the measurement of differential acceleration between the nail and the contact surface, expressing the mechanical loading applied to the finger. Figure 6 (top) shows the accelerations of finger deformations for three participants. Each plot is the average of ten trials under the loading only condition (Condition 1) after low-pass filtering at 200-Hz.

The middle panel shows the bulk deformation after two steps of integration of the differential acceleration signal. There is clearly some amount of drift in the estimates due to imperfect calibration of the accelerometers, yet the profile reveal quite different finger mechanics from one subject to another. The particular examples were selected to show the great differences in bulk deformation of three fingers. The relation to the tangential load profiles shown in the bottom panel shows complex dynamics that could be the result of combined nonlinear biomechanics and neural response.

Focusing on the initial portions of the responses, the finger pad deformation started approximately $15 \mathrm{~ms}$ later than the onset of the traction force. These times of onset were very similar among all the participants. Furthermore, the initial slopes of acceleration values were also comparable among them. They reach their first maximums around $30 \mathrm{~ms}$ later the onset of the pulling force. Then, they differed significantly among the participants. Similarly, the profiles 


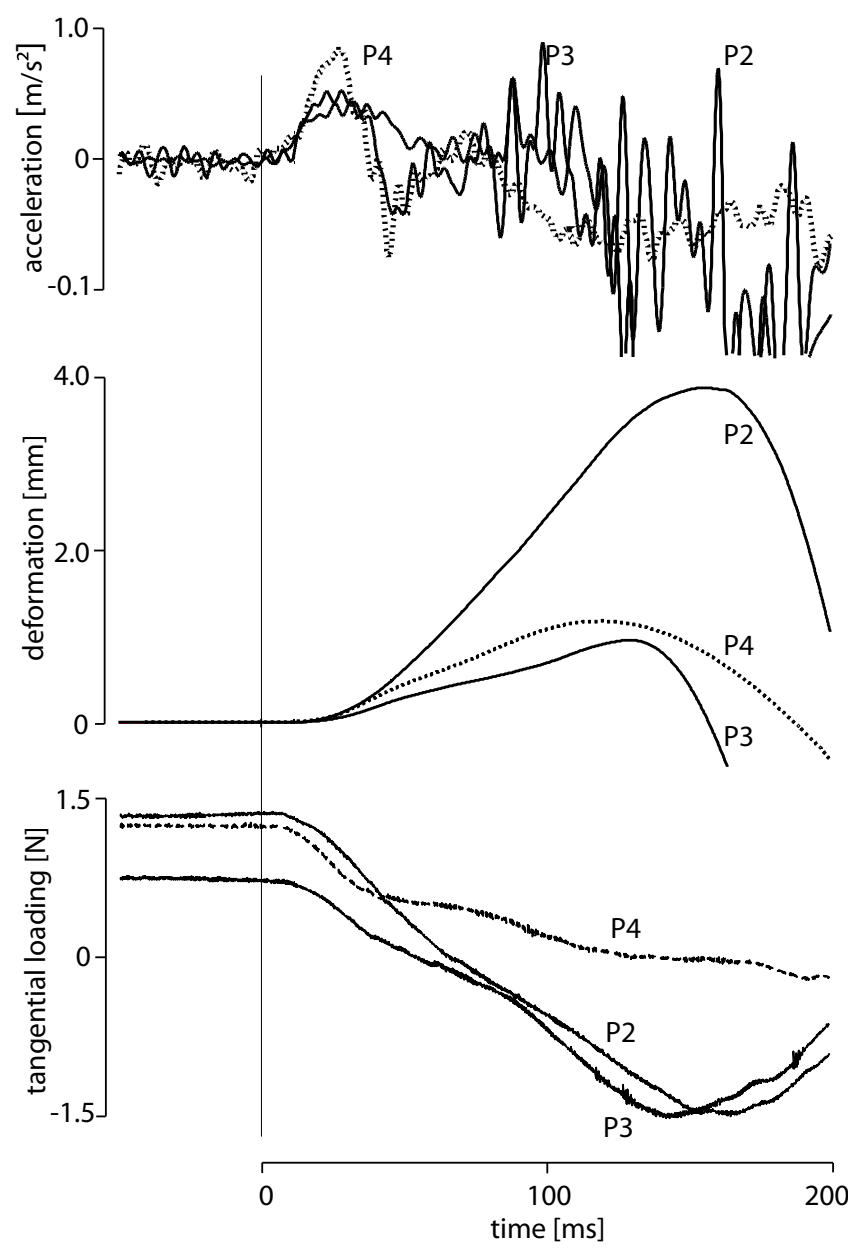

Figure 6: Finger pads' deformations and their tangential forces. Top) Acceleration of the deformations. Middle) Displacements. Bottom) Tangential forces.

of tangential forces were very similar for the first $30 \mathrm{~ms}$ for all the participants and differed later.

It can be surmised from these observations that the initial dynamics of the interaction are dominated by the tissue mechanics characterized by the typical nonlinear stiffening of the finger tissues undergoing large deformation $[13,18]$ and that this initial phase of $30 \mathrm{~ms}$ is followed by mechanical events precipitated by the neural response that can vary greatly among individuals. For example, one participant recorded a maximum pad deformation of approximately $4 \mathrm{~mm}$ on average, but for another participant this deformation was smaller than $1 \mathrm{~mm}$. The mechanics of the fingertip depend, not only in tangential loading, but also on normal loading. Another source of differences were the grip forces before and after application of the disturbances ([base-line $\rightarrow$ increase of grip force] $=[3.3 \rightarrow 2.8]$, [3.8 $\rightarrow 15.7],[5.3 \rightarrow 8.3] \mathrm{N}$ for $\mathrm{P} 2, \mathrm{P} 3$, and $\mathrm{P} 4$, respectively) which could affect the biomechanical as well as the neural response. P2's grip-force increases were the lowest but the finger deformation was the largest among the participants. P3's grip-force increases were greatest but the finger skin deformations were lowest. It is however risky to draw any conclusion from this apparent correlation.

\section{Discussion and Conclusion}

Under our experimental conditions, the response latencies to a sudden traction loading pulling force were $139 \pm 30 \mathrm{~ms}$ on average (Condition 1). Our definition of the latency is conservative and la- tencies and the neural response to disturbance is likely to be much shorter since the mechanics are expected to lag the neural activation of the muscles. Give or take the differences in the definitions of what latency is, our results are in line with those of the literature $[2,7,6]$. These value are also known to vary in a wide range (70 to $170 \mathrm{~ms}$ ) with the nature of the cueing given by particular types of disturbances owing to the differential engagements of spinal and supraspinal sensorimotor control loops.

Briefly, $50 \mathrm{~ms}$ vibrotactile cueing could influence the onset of grip force adjustment of a sudden unexpected change of load by $23 \mathrm{~ms}$ on average from a baseline value of $139 \mathrm{~ms}$. This can be compared to the $229 \pm 97 \mathrm{~ms}$ response to the same vibrotactile cue presented in the absence of loading disturbance, which can be interpreted as a conscious reaction time to a vibrotactile cue or to the $172 \pm 55 \mathrm{~ms}$ of reaction time when the cue was anticipated. It is thus permitted to conclude that the participant fast behavior was not the result of a conscious reaction and that the vibrotactile cues did modify their reflex reaction.

Mrotek et al. [11] pointed out the contributions of preparatory activities to grip force adjustment. They compared the latencies of grip force adjustment obtained during holding to those during lifting-object phases. They reported that the latencies in latter phase were smaller. During the lifting phase, tactile activities of finger pads are comparatively higher owing to the presence of inertial forces, raising the mechanical loading and thus the level of sensory inputs. They also found that the lifting phase led to larger increases in the grip forces. Here, we stimulated the tactile receptors using vibrotactile cue before the onset of the perturbative loading. The latency of grip force adjustment decreased and the grip forces has a tendency to increase. These effects could be related.

Finally, we did not find any significant differences between the two frequencies: 250 and $100 \mathrm{~Hz}$. However, this does not lead to a conclusion that there is no specification of mechanoreceptor classes because the frequency tuning method does not always insure the activation of specific receptor class.

We also could estimate the finger pad deformation in the course of a sudden disturbance load. Interestingly, the initial phase of mechanical response did not vary significantly among the few individuals that we tested, that is during the first few tens of milliseconds after the onset of the disturbance. On the other hand, the mechanical responses vary visibly among individuals, suggesting different sensorimotor strategic neural responses to similar inputs.

\section{ACKNOWLEDGEMENTS}

The authors wish to thank Dr. Alexander Terekhov for discussing the experimental procedures and results, Sheng-Chao Wong, and Rafał Pijewski for mechanical and electrical design of the equipment. The authors also thank Mark Farrington for input on the experimental platform. This work has been supported by the Technical Committee on Haptics, Student Exchange Program. MW and VH acknowledge the support of European Research Council, Advanced Grant PATCH, agreement No. 247300.

\section{REFERENCES}

[1] A.-S. Augurelle, A. M. Smith, T. Lejeune, and J.-L. Thonnard. Importance of cutaneous feedback in maintaining a secure grip during manipulation of hand-held objects. Journal of Neurophysiology, 89(2):665-671, 2003.

[2] K. J. Cole and J. H. Abbs. Grip force adjustments evoked by load force perturbations of a grasped object. Journal of Neurophysiology, 60(4):1513-1522, 1988.

[3] J. R. Flanagan and A. M. Wing. The role of internal models in motion planning and control: Evidence from grip force adjustments during movements of hand-held loads. Journal of Neuroscience, 17(4):15191528, 1997. 
[4] V. Hayward, P. Gregorio, O. Astley, S. Greenish, M. Doyon, L. Lessard, J. McDougall, I. Sinclair, S. Boelen, X. Chen, J.-P. Demers, J. Poulin, I, Benguigui, N. Almey, B. Makuc, and X. Zhang. Freedom-7: A high fidelity seven axis haptic device with application to surgical training. In A. Casals and A. T. de Almeida, editors, $E x$ perimental Robotics V, volume 232 of Lecture Notes in Control and Information Science, pages 445-456. Springer Verlag, 1998.

[5] R. S. Johansson, C. Häger, and L. Bäckström. Somatosensory control of precision grip during unpredictable pulling loads. III. Impairments during digital anaesthesia. Experimental Brain Research, 89:204-213, 1992.

[6] R. S. Johansson, C. Häger, and R. Riso. Somatosensory control of precision grip during unpredictable pulling loads. II. Changes in load force rate. Experimental Brain Research, 89:192-203, 1992.

[7] R. S. Johansson and G. Westling. Roles of glabrous skin receptors and sensorimotor memory in automatic control of precision grip when lifting rougher or more slippery objects. Experimental Brain Research, 56:550-564, 1984

[8] P. J. Kyberd and P. H. Chappell. Characterization of an optical and acoustic touch and slip sensor for autonomous manipulation. Measurement Science and Technology, 3(10):969, 1992.

[9] P. J. Kyberd, M. Evans, and S. Winkel. An intelligent anthropomorphic hand, with automatic grasp. Robotica, 16(5):531-536, 1998.

[10] T. Maeno, S. Hiromitsu, and T. Kawai. Control of grasping force by detecting sticwslip distribution at the curved surface of an elastic finger. Proceedings of IEEE International conference on robotics and automation, pages 3895-3900, 2000.

[11] L. A. Mrotek, B. A. Hart, P. K. Schot, and L. Fennigkoh. Grip responses to object load perturbations are stimulus and phase sensitive. Experimental Brain Research, 155:413-420, 2004.

[12] M. Nakamoto, M. Konyo, T. Maeno, and S. Tadokoro. Reflective grasp force control of humans induced by distributed vibration stimuli on finger skin with ICPF actuators. Proceedings of IEEE International conference on robotics and automation, pages 3899-3904, 2006.

[13] N. Nakazawa, R. Ikeura, and H. Inooka. Characteristics of human fingertips in the shearing direction. Biological Cybernetics, 82:207214, 2000.

[14] D. A. Nowak, J. Hermsdörfer, G. S, J. Philipp, L. M., and N. Mai. The effects of digital anaesthesia on predictive grip force adjustments during vertical movements of a grasped object. European Journal of Neuroscience, 14:756-762, 2001.

[15] T. Sakurai, M. Konyo, S. Okamoto, and S. Tadokoro. Research of conditions of stimulus for inducing grasping force control reflex. Proceedings of the 2000 IEEE/SICE International Symposium on System Integration, pages 408-413, 2010.

[16] M. R. Tremblay and M. R. Cutkosky. Estimation of friction using incipient slip sensing during a manipulation task. Proceedings of the 1993 IEEE International Confernce on Robotics and Automation, 1:429-434, 1993.

[17] J. Ueda, A. Ikeda, and T. Ogasawara. Grip-force control of an elastic object by vision-based slip-margin feedback during the incipient slip. IEEE Transactions on Robotics, 21(6):1139-1147, 2005.

[18] Q. Wang and V. Hayward. In vivo biomechanics of the fingerpad skin under local tangential traction. Journal of Biomechanics, 40(4):851860, 2007.

[19] M. Wiertlewski, S. Endo, A. Wing, and V. Hayward. Slip-induced vibration influences the grip reflex: A pilot study. Proceedings of the 2013 IEEE World Haptics Conference, 2013.

[20] Y. Yamada, H. Morita, and Y. Umetani. Vibrotactile sensor generating impulsive signals for distinguishing only slipping states. Proceedings of the 1999 IEEE/RSJ International Conference on Intelligent Robots and Systems, pages 844-850, 1999. 